\title{
Assessing the Effects of Air Abrasion with Aluminum Oxide or Glass Beads to Zirconia on the Bond Strength of Cement
}

\author{
Kibrom Mehari ${ }^{1}$, Allan S Parke ${ }^{2}$, Francisco F Gallardo ${ }^{3}$, Kraig S Vandewalle ${ }^{4}$
}

\begin{abstract}
Aim: The purpose of this study was to evaluate the effects of air abrasion with aluminum oxide or glass beads to three types of zirconia containing various levels of cubic crystalline phases (3Y-TZP, Katana ML; 4Y-PSZ, Katana STML; and 5Y-PSZ, Katana UTML, Noritake) on the shear bond strength of resin cement.

Materials and methods: Thirty block specimens $(8 \times 8 \times 3.5 \mathrm{~mm})$ were milled out of each zirconia material and mounted in plastic pipe. Ten specimens of each of the zirconia materials were air-abraded using $50 \mu \mathrm{m}$ aluminum oxide particles, ten specimens were abraded using 80 $\mu \mathrm{m}$ glass beads, and ten specimens served as a control and received no surface treatment. A zirconia primer was applied to the surface of the zirconia specimens. Composite disks were bonded using a resin cement and light-cured. The specimens were stored in $37^{\circ} \mathrm{C}$ distilled water for 24 hours and thermocycled for 2,500 cycles. The specimens were loaded in shear on a universal testing machine. Data were analyzed with one-way and two-way ANOVAs and Tukey's post hoc tests $(\alpha=0.05)$.

Results: A significant difference in shear bond strength was found based on the surface treatment $(p<0.001)$, but not on the type of zirconia $(p=0.132)$.

Conclusion: Air abrasion with glass beads or no surface treatment resulted in significantly lower bond strength of the resin cement to all three zirconia types compared to air abrasion with aluminum oxide.

Clinical significance: Although air abrasion with aluminum oxide may reportedly be more likely to weaken cubic-containing zirconia compared to air abrasion with glass beads, the use of aluminum oxide results in greater bond strength of the resin cement.

Keywords: Bond strength, Cement, Surface treatment, Zirconia.

The Journal of Contemporary Dental Practice (2020): 10.5005/jp-journals-10024-2879
\end{abstract}

\section{INTRODUCTION}

Zirconia is a metastable material that can exist in various crystalline phases. Three types of which have been utilized for dentistry: monoclinic, tetragonal, and cubic. The first version of zirconia employed in dentistry was a form comprised of the high-strength tetragonal crystalline phase. ${ }^{1}$ At room temperature, zirconia exists in the weaker monoclinic crystalline form. However, small amounts of oxides or dopants like yttrium oxide $\left(\mathrm{Y}_{2} \mathrm{O}_{3}\right)$ are added to stabilize it in the tetragonal crystalline form. ${ }^{1} \mathrm{Y}_{2} \mathrm{O}_{3}$ is added to the purified zirconia powder to stabilize the tetragonal phase and prevent it from transforming to the weaker monoclinic phase with sintering. ${ }^{1}$ Of all the restorative ceramics, $\mathrm{Y}_{2} \mathrm{O}_{3}$-stabilized tetragonal zirconia polycrystal (Y-TZP) is the most clinically durable. ${ }^{2}$

However, current Y-TZP tetragonal zirconia materials on the market lack the esthetics of competitive glass-ceramics and are therefore somewhat restricted to the posterior region or frameworks. ${ }^{2}$ To increase the translucency, new formulations of zirconia oxide were developed. Formulating new translucent dental zirconia materials involved increasing the $\mathrm{Y}_{2} \mathrm{O}_{3}$ content, which introduced the cubic phase along with the metastable tetragonal phase in the resulting zirconia oxide materials. ${ }^{3}$ The presence of specific percentages of tetragonal and cubic phases defines the mechanical and physical properties of the zirconia. Using a higher $\mathrm{Y}_{2} \mathrm{O}_{3}$ content produces a partially stabilized zirconia (PSZ) with greater cubic content. For example, the amount of $\mathrm{Y}_{2} \mathrm{O}_{3}$ dopant in molar concentration that is used in zirconia is abbreviated as 3Y-TZP for $3 \mathrm{~mol} \% \mathrm{Y}_{2} \mathrm{O}_{3}, 4 \mathrm{Y}$-PSZ for $4 \mathrm{~mol} \% \mathrm{Y}_{2} \mathrm{O}_{3}$, or $5 \mathrm{Y}$-PSZ for 5 mol\% $\mathrm{Y}_{2} \mathrm{O}_{3} .{ }^{3}$ In these new zirconia materials, the quantity of the cubic phase increases from $15 \%$ in 3 Y-TZP materials to approximately 25 vol\% in 4 Y-PSZ materials

\begin{abstract}
${ }^{1-4}$ Department of General Dentistry, Advanced Education in General Dentistry Residency, Wilford Hall Ambulatory Surgical Center, Dunn Dental Clinic, San Antonio-Lackland, Texas, USA; Uniformed Services University of the Health Sciences, Bethesda, Maryland, USA
\end{abstract}

Corresponding Author: Kraig S Vandewalle, Department of General Dentistry, Advanced Education in General Dentistry Residency, Wilford Hall Ambulatory Surgical Center, Dunn Dental Clinic, San AntonioLackland, Texas, USA; Uniformed Services University of the Health Sciences, Bethesda, Maryland, USA, Phone: +210 292-0760, e-mail: kraig.s.vandewalle.civ@mail.mil

How to cite this article: Mehari K, Parke AS, Gallardo FF, et al. Assessing the Effects of Air Abrasion with Aluminum Oxide or Glass Beads to Zirconia on the Bond Strength of Cement. J Contemp Dent Pract 2020;21(7):713-717.

Source of support: 59th Medical Wing, Joint Base San Antonio, Lackland, Texas, USA

Conflict of interest: None

and up to 50 vol\% in 5Y-PSZ materials. This increased cubic phase improves the translucency of the zirconia materials compared to traditional 3Y-TZP zirconia materials but diminishes toughness and strength. ${ }^{4}$ 3Y-TZP has a high fracture toughness from 5 to $10 \mathrm{MPa}$ $\mathrm{m}^{1} / 2$ and flexural strength of $900-1,400 \mathrm{MPa} .{ }^{3} 4 \mathrm{Y}-\mathrm{PSZ}$ has a fracture toughness of 2.5-3.5 MPa m $1 / 2$ and flexural strength of $600-900 \mathrm{MPa}$, and $5 Y$-PSZ has a fracture toughness of 2.2 to $4 \mathrm{MPa} \mathrm{m}^{1} / 2$ and flexural strength of 700-800 MPa. ${ }^{4}$

One of the unique things about zirconia is its increased ability to resist fracture. This property is called transformation toughness. The transformation of zirconia's metastable tetragonal phase to the monoclinic phase allows for this toughening, which increases a 
zirconia restoration's resistance to fracture. ${ }^{5}$ A certain disadvantage of $4 Y-P S Z$ and $5 Y-P S Z$ is the lower fracture toughness compared to 3Y-TZP. The translucent materials have smaller amounts of tetragonal phase (75\% in $4 \mathrm{Y}-\mathrm{PSZ}$ and $\sim 50 \%$ in $5 \mathrm{Y}$-PSZ), leading to a reduced possibility of tetragonal to monoclinic transformation and therefore less transformation toughening. ${ }^{4}$

Zirconia restorations are considered cementable with conventional cements due to their high flexural strength, but may need to be bonded if mechanical retention is limited. ${ }^{6}$ Bonding to 3 Y-TZP has been studied with recommendations that aluminum oxide abrasion and adhesive monomer application be utilized for more predictable long-term bonding. Concerns have been raised regarding surface damage after air-abrading 3Y-TZP with aluminum oxide particles. ${ }^{7,8}$ However, the results from a subgroup analysis demonstrated that air abrasion may actually improve the mechanical strength of 3Y-TZP compared to non-abraded specimen, irrespective of the air pressure or duration. ${ }^{9}$ No strength decrease was observed even after longer abrasion times. ${ }^{7}$ The monomer 10-methacryloyloxy-decyl dihydrogen phosphate (10MDP) was originally designed to bond to metal oxides and its use has been extended to oxide ceramics. 10-MDP-containing resin cements or primers seem to be the most successful due to the chemical interaction between the hydroxyl groups of the passive zirconia surface and the phosphate ester group of the 10-MDP. ${ }^{10}$ A recent study examined the bond strength between an adhesive resin cement and cubic-containing zirconia and found it was similar to traditional tetragonal zirconia. ${ }^{11}$ However, the weaker cubic-containing zirconia materials may require a different bonding strategy when using air abrasion.

Cubic-containing zirconia may not undergo transformation toughening and, therefore, may be more susceptible to mechanical damage with aluminum oxide air abrasion. The surface treatment with glass beads instead of aluminum oxide has been suggested for cubic-containing zirconia and does not seem to result in a strength degradation. ${ }^{12}$ A recent study found that the flexural strength of esthetic cubic-containing zirconia was adversely affected by aluminum oxide air abrasion and to a less extent by glass beads. ${ }^{13}$ Although glass beads may be less likely to weaken the cubic-containing zirconia, it may not prepare the surface as well as aluminum oxide and therefore, it may result in lower bond strength of resin cement.

The purpose of this study was to evaluate the effects of aluminum oxide and glass bead air abrasion to 3Y-TZP, 4Y-PSZ, and 5Y-PSZ zirconia materials on the shear bond strength of a resin cement. The null hypotheses tested were that there would be no difference in shear bond strength of a resin cement based on (1) surface treatment or (2) type of zirconia.

\section{Materials and Methods}

This original research study was conducted at the United States Air Force Postgraduate Dental School at Joint Base San Antonio (JBSA), Lackland, TX, USA. The Institutional Review Board at Wilford Hall Ambulatory Surgical Center, JBSA, Lackland, TX, USA, approved the protocol \#FWH20190051N. The following materials were tested: 3Y-TZP multilayered (Katana ML, Shade 1.5-2), 4Y-PSZ supertranslucent multilayered (Katana STML, Shade A2), and 5Y-PSZ ultra-translucent multilayered zirconia (Katana UTML, Shade A2, Kuraray Dental, Houston, TX, USA). Aluminum oxide particles and glass beads were used for air particle surface treatment. Ninety specimens were created for shear bond strength testing. Ten specimens per each of the three ceramic materials were created and subjected to three different surface treatments (aluminum oxide air abrasion, glass-bead air abrasion, or no surface treatment).

Thirty block specimens $(8 \times 8 \times 3.5 \mathrm{~mm})$ were designed and milled out of each of the zirconia materials. The blocks were designed using DS SolidWorks software (SolidWorks, Waltham, MA, USA), and the file was imported into Sum 3D, iCAM V5 milling software (I-Mes, iCore, Eiterfeld, Germany). A CAM (computeraided manufacturing) machine (I-Mes iCore 450i) was used to mill the zirconia blocks out of the zirconia blanks. After sintering the blocks in a furnace (Programat S1 1600, Ivoclar Vivadent, Amherst, NY, USA), the zirconia blocks were mounted in a plastic pipe using resin (Vitacrilic, Fricke International, Streamwood, IL, USA). The top surface of the block specimen received either air abrasion with aluminum oxide, glass beads, or no surface treatment. The blocks from the aluminum oxide group were air-abraded (Basic Quattro IS, Renfert, Chicago, IL, USA) using $50 \mu$ m aluminum oxide (Korox, BEGO, Bremen, Germany) at 2.0 bar at a distance of $10 \mathrm{~mm}$ for 10 seconds using a vinyl polysiloxane jig to standardize distances. Similarly, the blocks from the glass-bead group were air-abraded using $80 \mu \mathrm{m}$ glass beads (Perlablast Micro, BEGO, Bremen, Germany) (Table 1). A control group received no surface treatment. Then, the blocks were steam cleaned (i700B, Reliable, Toronto, Ontario).

Ninety composite resin disks (Z250 3M ESPE, St Paul, MN, USA) were produced using a custom-made metal split mold ( $4.0 \mathrm{~mm}$ internal diameter and $2.0 \mathrm{~mm}$ thickness) positioned between two glass slabs covered with transparent polyester films. Light polymerization of the composite was performed for 40 seconds on the top surface and two diametrically opposed sides of the resin disks after removal from the mold (total of 120 seconds) using the Bluephase 20i (Ivoclar Vivadent) light-curing unit. The irradiance of the curing light was determined with a radiometer (LED Radiometer, Kerr, Orange, CA, USA) to verify the irradiance levels of at least $1,200 \mathrm{~mW} / \mathrm{cm}^{2}$. A thin layer of phosphate-based 10-MDP primer (Z-Prime Plus, Bisco, Schaumburg, IL, USA) was applied to the top surface of the zirconia block according to the manufacturer's instructions. A dual-cure resin cement (NX3, Kerr) was used to bond to the prepared composite cylinders to the zirconia blocks. A thin layer of the mixed cement was applied and distributed to the bonding surface. A $100 \mathrm{~g}$ calibration weight was placed on the composite cylinders to ensure a standardized film thickness of cement. After excess cement was removed, the cement

Table 1: Ceramic and surface treatment materials utilized in this study

\begin{tabular}{|c|c|c|c|}
\hline & Name & Type & Company \\
\hline \multirow[t]{3}{*}{$\begin{array}{l}\text { Ceramic } \\
\text { material }\end{array}$} & $\begin{array}{l}\text { Katana ML, } \\
\text { Shade } 1.5-2\end{array}$ & $\begin{array}{l}3 \text { mol\% yttria- } \\
\text { stabilized } \\
\text { tetragonal } \\
\text { zirconia } \\
\text { polycrystal } \\
\text { (3Y-TZP) }\end{array}$ & $\begin{array}{l}\text { Kuraray } \\
\text { Noritake, } \\
\text { Kurashiki, } \\
\text { Okayama, } \\
\text { Japan }\end{array}$ \\
\hline & $\begin{array}{l}\text { Katana } \\
\text { STML, Shade } \\
\text { A2 }\end{array}$ & $\begin{array}{l}4 \text { mol\% partially } \\
\text { stabilized zirconia } \\
\text { (4Y-PSZ) }\end{array}$ & \\
\hline & $\begin{array}{l}\text { Katana } \\
\text { UTML, Shade } \\
\text { A2 }\end{array}$ & $\begin{array}{l}5 \text { mol\% partially } \\
\text { stabilized zirconia } \\
\text { (5Y-PSZ) }\end{array}$ & \\
\hline \multirow{2}{*}{$\begin{array}{l}\text { Mechanical } \\
\text { surface } \\
\text { treatment }\end{array}$} & Korox & $\begin{array}{l}50 \mu \mathrm{m} \text { aluminum } \\
\text { oxide particles }\end{array}$ & $\begin{array}{l}\text { BEGO, Bremen, } \\
\text { Germany }\end{array}$ \\
\hline & $\begin{array}{l}\text { Perlablast } \\
\text { Micro }\end{array}$ & $\begin{array}{l}80 \mu \mathrm{m} \text { glass } \\
\text { beads }\end{array}$ & \\
\hline
\end{tabular}


was cured in four equidistant positions for 40 seconds each using the curing light as before.

The specimens were stored in $37^{\circ} \mathrm{C}$ distilled water in a lab oven (Model 20GC, Quincy Lab, Chicago, IL, USA) for 24 hours and then thermocycled in distilled water for 2,500 cycles at $5^{\circ} \mathrm{C}$ and $55^{\circ} \mathrm{C}$ with a dwell time of 30 seconds at each temperature (Sabri Dental Enterprises Inc, Downers Grove, IL, USA). The specimens were loaded perpendicularly with a blade-shaped probe in a universal testing machine (Model 5943, Instron, Norwood, MA, USA) using a crosshead speed of $1.0 \mathrm{~mm} /$ minute until failure. Shear bond strength values in megapascals ( $\mathrm{MPa}$ ) were calculated from the peak load of failure divided by the specimen surface area. A mean and standard deviation were determined per group. The data were submitted first to a Shapiro-Wilk test and found to be normally distributed. The data were then analyzed with a two-way ANOVA and Tukey's post hoc test evaluating the effect of zirconia material (3 levels) or surface treatment (3 levels) on shear bond strength ( $\alpha$ $=0.05$ ). Additionally, the data were analyzed with multiple one-way ANOVAs per zirconia material or surface treatment.

Following testing, each shear bond strength specimen was examined using a light microscope (SMZ-1B, Nikon, Melville, NY, USA) at $10 \times$ magnification to determine failure mode as either: (1) adhesive fracture at the resin cement/zirconia interface, (2) cohesive fracture in resin cement, (3) mixed (combined adhesive and cohesive) in resin cement or zirconia, or (4) cohesive fracture in zirconia. Representative specimens from each group were imaged with a scanning electron microscope (TM 3000, Hitachi, Tarrytown, NY) at 40× magnification. Statistical analysis was performed using SPSS 25 (IBM/SPSS, Chicago, IL, USA).

\section{Results}

A significant difference in shear bond strength of the resin cement was found based on the surface treatment $(p<0.001)$, but not on zirconia type ( $p=0.132)$, with no significant interaction ( $p=0.98$ ). Air abrasion with aluminum oxide created significantly greater bond strength of the resin cement to all zirconia materials compared to air abrasion with glass beads or no surface treatment. No significant difference in shear bond strength of the resin cement was found between the use of glass beads and no surface treatment (Table 2). There was no significant difference in shear bond strength between any of the three zirconia materials based on the surface treatment. More mixed failures were observed with aluminum oxide air abrasion as shown in Figure 1. Representative failure modes at $40 \times$ magnification are shown in Figure 2.

\section{Discussion}

The survival of zirconia restorations with less retentive preparations relies on, among other aspects, the durability of the bonded

Table 2: Shear bond strength of the resin cement to various zirconia types after surface treatments with no treatment (control), glass beads, or aluminum oxide. Groups with the same upper case letter per row or lower case letter per column are not significantly different $(p>0.05)$

\begin{tabular}{lrrr}
\hline \multirow{2}{*}{$\begin{array}{l}\text { Surface treat- } \\
\text { ment }\end{array}$} & \multicolumn{3}{c}{ Shear bond strength MPa (Std dev) } \\
\cline { 2 - 4 } Control & \multicolumn{1}{c}{ Katana ML } & \multicolumn{1}{c}{ Katana STML } & \multicolumn{1}{c}{ Katana UTML } \\
\hline Glass beads & $5.9(1.7) \mathrm{Aa}$ & $5.1(1.3) \mathrm{Aa}$ & $6.4(1.4) \mathrm{Aa}$ \\
Al oxide & $13.4(3.8) \mathrm{Ab}$ & $11.8(1.0) \mathrm{Aa}$ & $5.6(1.1) \mathrm{Aa}$ \\
\hline
\end{tabular}

interfaces. ${ }^{2}$ Zirconia is a densely sintered material exhibiting high hardness, and thus, roughening the surface of the ceramic may be more difficult. ${ }^{14,15}$ Zirconia does not contain silica-glass particles like traditional glass-ceramics allowing an etch with hydrofluoric acid and bond with silane to the intaglio surface. Therefore, it may require additional surface treatment (e.g., air abrasion) and surface primers (e.g., 10-MDP) for less retentive preparations. Air abrasion may clean the ceramic surface, remove impurities, and increase surface roughness, bond strength, surface energy, and wettability. ${ }^{2}$ Air abrasion has been reported to both increase and decrease the mechanical strength of 3Y-TZP zirconia materials and to promote varied percentages of phase transformation. ${ }^{16-18}$ These contradictory findings may result from the diverse protocols used, with variation in particle size and pressure, as well as the lack of aging conditions. However, global results from a meta-analysis showed that the use of surface conditioning procedures based on the airborne-particle abrasion protocols improved the flexural strength of 3Y-TZP. ${ }^{7}$ The force of the particles on the 3Y-TZP surface can lead to microcracks and plastic deformation, thus decreasing the strength of the ceramic. ${ }^{16,19}$ However, small cracks, flaws, and defects that could lead to fracture appear to remain confined within the transformation layer, where they are probably healed by the $4 \%$ volume increase in the grains during the phase transformation. ${ }^{20}$ However, a recent study found that the flexural strength of $4 Y-P S Z$ and 5Y-PSZ cubic-containing zirconia materials was adversely affected by aluminum oxide air abrasion and to a less extent by the use of glass beads. ${ }^{13}$

In this study, air abrasion with aluminum oxide created greater bond strength of the resin cement to all zirconia materials compared to air abrasion with glass beads or no surface treatment. Therefore, the first null hypothesis was rejected. The use of the glass beads resulted in a zirconia surface that was similar in retention to no surface treatment but less retentive than the use of the aluminum oxide particles. Although glass beads may be less likely to weaken the cubic-containing zirconia as found by Cowen et al., the results of this study suggest that it may not prepare the surface as well as aluminum oxide and therefore lower the bond strength to resin cement. ${ }^{13}$ Similar to this study, a recent study by Le et al. found that aluminum oxide air abrasion increased the bond strength significantly for both conventional and translucent zirconia. ${ }^{11}$ So, until more studies are published comparing the effect of air abrasion using aluminum oxide or glass beads on the strength of cubiccontaining zirconia, the authors suggest that practitioners continue to air-abrade all types of zirconia (i.e., 3Y-TSP, 4Y-PSZ, and 5Y-PSZ) with aluminum oxide and use an adhesive monomer to increase bond strength in clinical cases with less retentive preparations. The selection of the type of zirconia to utilize clinically will depend on individual case selection. The stronger, but less esthetic 3Y-TZP zirconia materials may be selected for posterior restorations with heavier occlusion and the weaker, but more esthetic cubiccontaining zirconia materials may be considered for more anterior restorations in the esthetic zone. ${ }^{1,3}$

Although there were differences in cubic content and inherent strength properties of the three materials, there was not a significant difference in bond strength per surface treatment. Therefore, the second null hypothesis was not rejected. Air abrasion with aluminum oxide resulted in over twice the shear bond strength than the use of glass beads or no surface treatment-irrespective of material type. Additionally, more mixed failures were associated with aluminum oxide air abrasion compared to the use of glass 


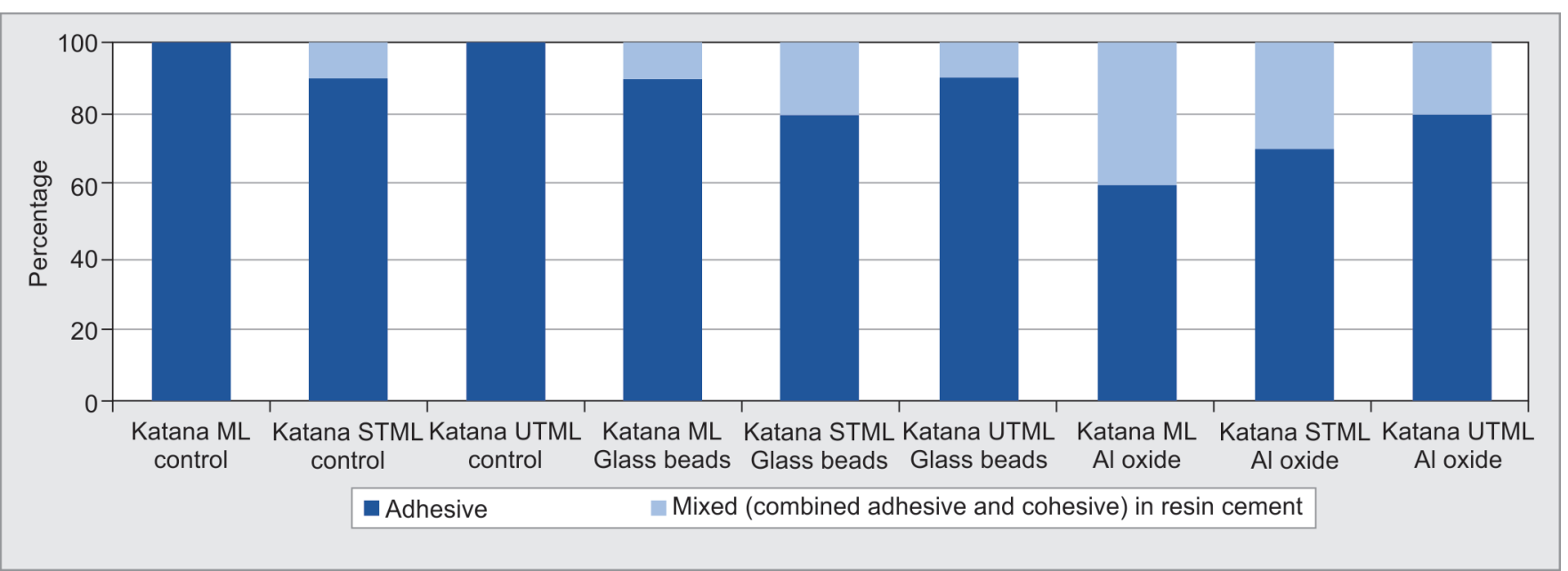

Fig. 1: Graph of failure modes of the resin cement to various zirconia types after surface treatment with no treatment (control), glass beads, or aluminum oxide. Failures were found to be adhesive or mixed and expressed as a percentage of all specimens per group
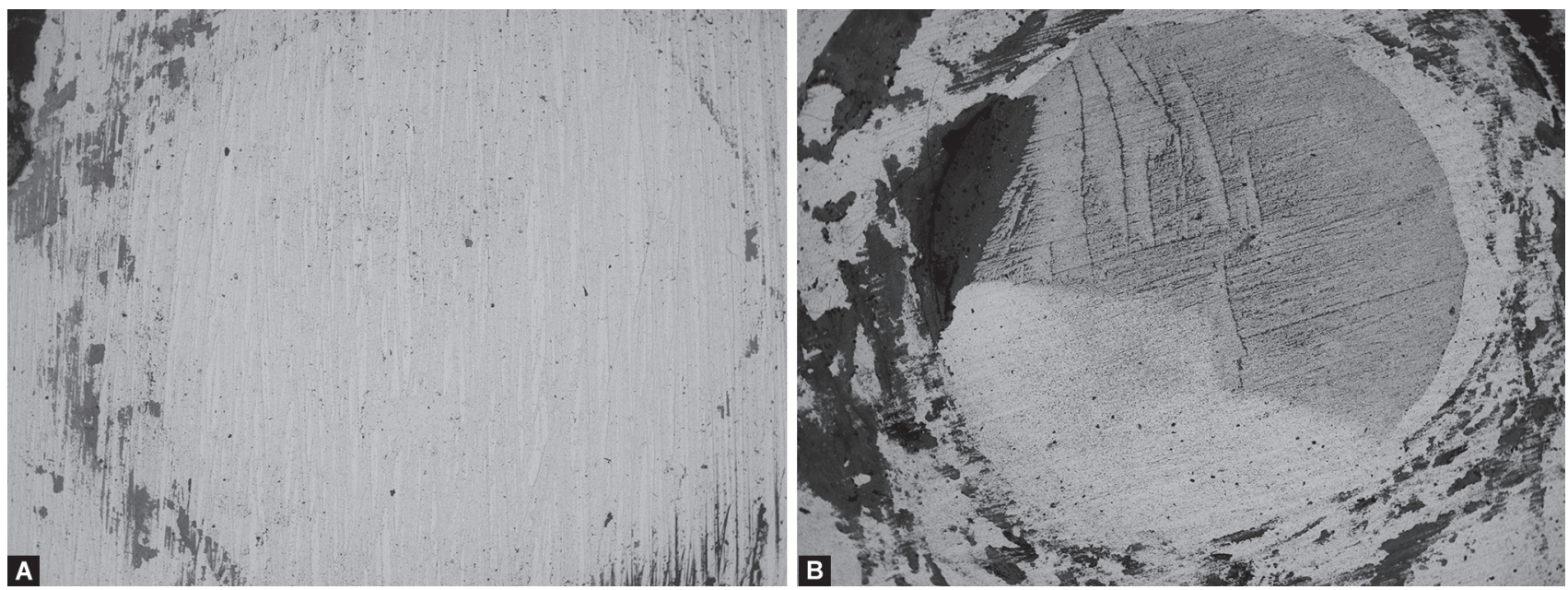

Figs $2 A$ and B: Representative failure modes at 40x magnification: (A) Primary failure through the adhesive interface as seen with many of the control (no surface treatment) and air abrasion with glass bead specimens; (B) Mixed fracture through the adhesive layer and cement was seen more commonly with the aluminum oxide air abrasion specimens

beads. Mixed failure modes are often associated with higher bond strength compared to purely adhesive-type failures, as was seen more readily with the use of glass beads and even more evident in the untreated control group. ${ }^{21}$

Limitations to this study include the use of only one type of cement and adhesive primer. Additionally, the effect of various sizes of aluminum oxide particles and glass beads should be investigated along with different levels of air pressure. Future clinical studies should examine the effects of air abrasion using aluminum oxide or glass beads on the intra-oral success of cubic-containing zirconia.

\section{Conclusion}

Air abrasion with glass beads or no surface treatment resulted in significantly lower bond strength of the resin cement to all three zirconia types compared to air abrasion with aluminum oxide.

\section{Disclaimer}

The views expressed are those of the authors and do not reflect the official views or policy of the Uniformed Services University,
Department of Defense, or its Components. The authors do not have any financial interest in the companies whose materials are discussed in this abstract.

\section{References}

1. McLaren EA, Lawson N, Choi J, et al. New high-translucent cubicphase-containing zirconia: clinical and laboratory considerations and the effect of air abrasion on strength. Compend Contin Educ Dent 2017;38(6):e13-e16.

2. Miyazaki T, Nakamura T, Matsumura $H$, et al. Current status of zirconia restoration. J Prosthodont Res 2013;57(4):236-261. DOI: 10.1016/j. jpor.2013.09.001.

3. Zhang Y, Lawn BR. Novel zirconia materials in dentistry. J Dent Res 2018;97(2):140-147. DOI: 10.1177/0022034517737483.

4. Zhang F, Inokoshi M, Batuk M, et al. Strength, toughness and aging stability of highly-translucent Y-TZP ceramics for dental restorations. Dent Mater 2016;32(12):e327-e337. DOI: 10.1016/j.dental.2016. 09.025 .

5. Denry I, Kelly JR. State of the art of zirconia for dental applications. Dent Mater 2008;24(3):299-307. DOI: 10.1016/j.dental.2007.05. 007. 
6. Blatz MB, Conejo J. The current state of chairside digital dentistry and materials. Dent Clin North Am 2019;63(2):175-197. DOI: 10.1016/ j.cden.2018.11.002.

7. Aurélio IL, Marchionatti AM, Montagner AF, et al. Does air particle abrasion affect the flexural strength and phase transformation of Y-TZP? A systematic review and meta-analysis. Dent Mater 2016;32(6):827-845. DOI: 10.1016/j.dental.2016.03.021.

8. Zhang $Y$, Lawn BR, Rekow ED, et al. Effect of sandblasting on the long-term performance of dental ceramics. J Biomed Mater Res B Appl Biomater 2004;71(2):381-386. DOI: 10.1002/jbm.b.30097.

9. Egilmez F, Ergun G, Cekic-Nagas I, et al. Factors affecting the mechanical behavior of Y-TZP. J Mech Behav Biomed Mater 2014;37:78-87. DOI: 10.1016/j.jmbbm.2014.05.013.

10. Papia $\mathrm{E}$, Larsson $\mathrm{C}$, du Toit $\mathrm{M}$, et al. Bonding between oxide ceramics and adhesive cement systems: a systematic review. J Biomed Mater Res B Appl Biomater 2014;102(2):395-413. DOI: 10.1002/jbm.b.33013.

11. Le M, Larsson C, Papia E. Bond strength between MDP-based cement and translucent zirconia. Dent Mater J 2019;38(3):480-489. DOI: 10.4012/dmj.2018-194.

12. McLaren EA, Burgess JO, Brucia J. Cubic containing zirconia (CUZR): adhesive cement or conventional cement - what are the indications? Compend Contin Educ Dent 2018;39(5):21-25.

13. Cowen M, Wains S, Montealegre J, et al. Esthetic zirconia flexural strength after blasting with glass or alumina. J Dent Res 98(Spec Iss):2476 (www.iadr.org).

14. Casucci A, Osorio E, Osorio R, et al. Influence of different surface treatments on surface zirconia frameworks. J Dent 2009;37(11): 891-897. DOI: 10.1016/j.jdent.2009.06.013.
15. Wang RR, Lu CL, Wang G, et al. Influence of cyclic loading on the fracture toughness and load bearing capacities of all-ceramic crowns. Int J Oral Sci 2014;6(2):99-104. DOI: 10.1038/ijos.2013.94.

16. Garcia Fonseca R, de Oliveira Abi-Rached F, dos Santos Nunes Reis JM, et al. Effect of particle size on the flexural strength and phase transformation of an airborne-particle abraded yttriastabilized tetragonal zirconia polycrystal ceramic. J Prosthet Den 2013;110(6):510-514. DOI: 10.1016/j.prosdent.2013.07.007.

17. Ozcan M, Melo RM, Souza RO, et al. Effect of air-particle abrasion protocols on the biaxial flexural strength, surface characteristics and phase transformation of zirconia after cyclic loading. J Mech Behav Biomed Mater 2013;20:19-28. DOI: 10.1016/j.jmbbm.2013. 01.005 .

18. Souza RO, Valandro LF, Melo RM, et al. Air-particle abrasion on zirconia ceramic using different protocols: effects on biaxial flexural strength after cyclic loading, phase transformation and surface topography. J Mech Behav Biomed Mater 2013;26:155-163. DOI: 10.1016/j.jmbbm.2013.04.018.

19. Wang $\mathrm{H}$, Aboushelib MN, Feilzer AJ. Strength influencing variables on CAD/CAM zirconia frameworks. Dent Mater 2008;24(5):633-638. DOI: 10.1016/j.dental.2007.06.030.

20. Amaral M, Valandro LF, Bottino MA, et al. Low-temperature degradation of a Y-TZP ceramic after surface treatments. J Biomed Mater Res B Appl Biomater 2013;101(8):1387-1392. DOI: 10.1002/ jbm.b.32957.

21. Al-Salehi SK, Burke FJ. Methods used in dentin bonding tests: an analysis of 50 investigations on bond strength. Quintessence Int 1997;28:717-723. 